\title{
Hyper-Kähler Fourfolds Fibered by Elliptic Products
}

\author{
Ljudmila Kamenova
}

\begin{abstract}
Every fibration of a projective hyper-Kähler fourfold has fibers which are Abelian surfaces. In case the Abelian surface is a Jacobian of a genus two curve, these have been classified by Markushevich. We study those cases where the Abelian surface is a product of two elliptic curves, under some mild genericity hypotheses.
\end{abstract}

Keywords. Hyperkähler manifolds; Hilbert schemes; K3 surfaces; Lagrangian fibrations

2010 Mathematics Subject Classification. 14D06; 14E05; 53C26

\section{[Français]}

Titre. Variétés hyper-kähleriennes de dimension 4 fibrées par des produits elliptiques

Résumé. Toute fibration d'une variété hyper-kählerienne projective de dimension 4 a pour fibres des surfaces abéliennes. Dans le cas où la surface abélienne est la jacobienne d'une courbe de genre 2, elles ont été classifiées par Markushevich. Nous étudions le cas où la surface abélienne est un produit de deux courbes elliptiques, sous des hypothèses de généricité peu restrictives.

Received by the Editors on October 10, 2017, and in final form on May 8, 2018. Accepted on August 23, 2018.

Ljudmila Kamenova

Department of Mathematics, Room 3-115, Stony Brook University, Stony Brook, NY 11794-3651, USA

e-mail: kamenova@math.stonybrook.edu

Research partially supported by NSF Grants DMS-0111298 and DMS-0805594 


\section{Contents}

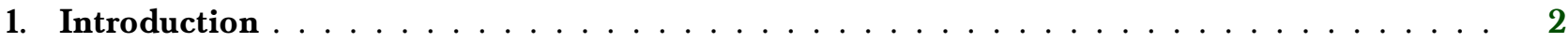

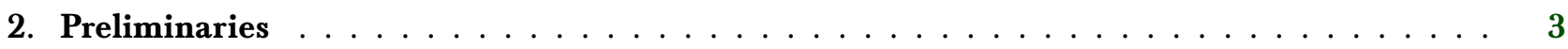

3. Proof of the Main Theorem $\ldots \ldots \ldots \ldots \ldots \ldots \ldots \ldots$

\section{Introduction}

Among projective complex manifolds with zero first Chern class, hyper-Kähler manifolds play a distinguished role, generalizing the class of K3 surfaces. In fact the hyper-Kähler manifolds of complex dimension two are precisely the K3 surfaces. In higher dimensions, dimension four and higher, few hyper-Kähler manifolds are known: two infinite classes introduced by Beauville ([2]) and two exceptional cases discovered by O'Grady ([12] and [13]). Nonetheless, even in the first case of hyper-Kähler fourfolds, we are still far from a classification or even a proof that hyper-Kähler fourfolds form a bounded family.

Just as elliptically fibered K3 surfaces are more amenable to study, also fibered hyper-Kähler manifolds over smooth bases are better understood. By works of Matsushita and Hwang, ([10,11] and [8]), every holomorphic fibration whose total space is a $2 n$-dimensional hyper-Kähler manifold has base manifold $\mathbb{P}^{n}$ and has general fiber an $n$-dimensional Abelian variety. A hyper-Kähler manifold fibered over $\mathbb{P}^{n}$ with general fiber an Abelian variety is called an Abelian fibration. Every Abelian fibered manifold is birational to a "Tate-Shafarevich twist" of an Abelian fibration with a section, and the set of these twists coming from a given Abelian fibration is classified by a well-studied Tate-Shafarevich group. Thus, from now on we assume that the Abelian fibration admits a section.

The principal case is when the fibers are principally polarized Abelian varieties. For hyper-Kähler fourfolds, the fiber will be a principally polarized Abelian surface. There are two types of such surfaces, depending on whether or not the polarizing divisor is irreducible or reducible, i.e., whether the Abelian surface is the Jacobian of a genus 2 curve or a product of elliptic curves. Markushevich classified the first case, when the Abelian surface is the Jacobian of a genus- 2 curve.

Theorem 1.1. (Markushevich, [9]) Every Abelian fibered irreducible holomorphic symplectic fourfold X which is the compactified relative Jacobian of a family of genus-two curves is birational to $\mathrm{Hilb}^{2}(S)$, for a K3 surface $S$ which is a double cover of $\mathbb{P}^{2}$ branched over a plane sextic.

Our goal is to classify those Abelian fibrations where the general fiber is a product of two elliptic curves, an elliptic product. These hyper-Kähler fourfolds can become "wild" when the fibration is allowed to become pathologically degenerate. In fact, we conjecture that these pathological degenerations never occur, at least for a general member of a deformation family. But, at the moment, we add our conjectured genericity conditions as hypotheses. Here is the main result of the paper.

Theorem 1.2. Consider a double cover map $f: \bar{V} \rightarrow \mathbb{P}^{2}$, where $\bar{V}$ is a normal variety, and a rational elliptic fibration $\pi: \mathcal{E} \rightarrow \bar{V}$ with a section $\sigma$, where $\mathcal{E}$ is projective. Let $D$ be the branch locus of $f, \tilde{D}=f^{-1}(D)$ and let $G$ be the discriminant locus of $\pi$. Let $i$ be the involution acting on $\bar{V}$ that interchanges the sheets of the double cover $f$ and let $i_{\mathcal{E}}$ be the induced involution on $\mathcal{E} \times_{\bar{V}} i^{*} \mathcal{E}$. Assume the following genericity conditions are satisfied:

(1) Over the general point of $G$, the fiber of $\pi$ is irreducible and semistable.

(2) The image $f(G)$ contains no singular point of $D$, and $G \cap i(G)$ is finite.

Assume that $\left(\mathcal{E} \times_{\bar{V}} i^{*} \mathcal{E}\right) / i_{\mathcal{E}} \rightarrow \mathbb{P}^{2}$ admits a resolution of singularities $p: X \rightarrow \mathbb{P}^{2}$ which is an Abelian fibration on a projective hyper-Kähler manifold $X$ with a section $\tau$. Then $\bar{V} \cong \mathbb{P}^{1} \times \mathbb{P}^{1}$ and $X$ is birational to Hilb ${ }^{2}(S)$ for a projective elliptic K3 surface $S$ with a section. 
Notice that for every projective, elliptic K3 surface $S$ with a section, $\operatorname{Hilb}^{2}(S)$ is a projective hyperKähler fourfold fibered by elliptic products over $\operatorname{Hilb}^{2}\left(\mathbb{P}^{1}\right)=\mathbb{P}^{2}$, and admits a section. In this example $\bar{V}=$ $\mathbb{P}^{1} \times \mathbb{P}^{1} \rightarrow \mathbb{P}^{2}$ is the standard double covering and the divisor $\mathcal{E} \subset \operatorname{Hilb}^{2}(S) \times_{\mathbb{P}^{2}} \bar{V}$ appears naturally. The question that we study inverts this construction under the assumptions (1) - (2) and we show that the only examples one obtains are birational to $\mathrm{Hilb}^{2}(S)$. There are countably many families of projective, elliptic $\mathrm{K} 3$ surfaces $S$, hence countably many families of elliptic product hyper-Kähler fourfolds. We conjecture that every sufficiently general elliptic product hyper-Kähler fourfold satisfies the genericity conditions. All such are deformation equivalent to one of the countably many families arising from projective, elliptic K3 surfaces.

\section{Preliminaries}

First we define our main objects of study, irreducible holomorphic symplectic manifolds or irreducible hyperKähler manifolds.

Definition 2.1. A compact complex Kähler manifold $X$ is called irreducible holomorphic symplectic if it is simply connected and if $H^{0}\left(X, \Omega_{X}^{2}\right)$ is spanned by an everywhere non-degenerate 2 -form $\sigma$.

The two-form $\sigma$ is everywhere non-degenerate if it induces an isomorphism $\mathcal{T}_{X} \rightarrow \Omega_{X}$. The last condition in the definition implies that $h^{2,0}(X)=h^{0,2}(X)=1$ and $K_{X} \cong \mathcal{O}_{X}$, i.e., $c_{1}(X)=0$.

Definition 2.2. A compact connected $4 n$-dimensional Riemannian manifold $(M, g)$ is called irreducible hyper-Kähler if its holonomy group is $\operatorname{Sp}(n)$.

As Huybrechts notes in [7], irreducible holomorphic symplectic manifolds with a fixed Kähler class are equivalent to irreducible hyper-Kähler manifolds with a fixed complex structure.

Definition 2.3. An Abelian fibration on a $2 n$-dimensional hyper-Kähler manifold $X$ is the structure of a fibration over $\mathbb{P}^{n}$ whose general fiber is a smooth abelian variety of dimension $n$.

This is a higher dimensional analogue of elliptic fibrations on K3 surfaces. Any fibration structure of a projective hyper-Kähler manifold is an Abelian fibration due to the following theorems by Matsushita [10, 11] and Hwang [8]:

Theorem 2.4. (Matsushita, $[10,11])$ For a projective irreducible holomorphic symplectic manifold $X$, let $f$ : $X \rightarrow B$ be a proper surjective morphism such that the general fiber $F$ is connected. Assume that $0<\operatorname{dim} B<\operatorname{dim} X$. Then

(1) F is an abelian variety;

(2) B is n-dimensional. If $B$ is smooth, then it has the same Hodge numbers as $\mathbb{P}^{n}$;

(3) the fibration is Lagrangian with respect to the holomorphic symplectic form.

Theorem 2.5. (Hwang, [8]) In the setting of Matsushita's theorem, if B is smooth, then B is biholomorphic to $\mathbb{P}^{n}$.

Fogarty gives the following description of the Hilbert schemes of complex surfaces.

Theorem 2.6. (Fogarty, [5]) For a nonsingular surface $X$ and $n \in \mathbb{N}$

(1) $\operatorname{Hilb}^{n}(X)$ is non-singular of dimension $2 n$, and

(2) $\pi: \operatorname{Hilb}^{n}(X) \rightarrow S^{n} X$ is a resolution of singularities, where $S^{n} X$ is the $n$-th symmetric product of $X$. 
Due to Beauville ([2]), Hilbert schemes of K3 surfaces give one of the standard series of examples of irreducible holomorphic symplectic manifolds. The other standard series of examples, generalized Kummer varieties (also due to Beauville), arise from Hilbert schemes of abelian surfaces. In [14] Wieneck proved that if $X$ is a hyper-Kähler manifold that admits a Lagrangian fibration, the polarization type of the fibration is a deformation invariant of the fibration. As Wieneck shows, for Lagrangian fibrations the "polarization type" of the fiber is independent from the choice of an integral Kähler form as long as it is obtained as the restriction from a Kähler class on the ambient hyperkähler manifold. Moreover, if $X$ is of $K 3^{[n]}$-type, then the polarization type is principal. If $X$ is of generalized Kummer type, then Wieneck showed that the polarization type of a Lagrangian fibration depends on the connected component of the moduli space, [15].

\section{Proof of the Main Theorem}

In this section we prove our main theorem.

Theorem 3.1. Consider a double cover map $f: \bar{V} \rightarrow \mathbb{P}^{2}$, where $\bar{V}$ is a normal variety, and a rational elliptic fibration $\pi: \mathcal{E} \rightarrow \bar{V}$ with a section $\sigma$, where $\mathcal{E}$ is projective. Let $D$ be the branch locus of $f, \tilde{D}=f^{-1}(D)$ and let $G$ be the discriminant locus of $\pi$. Let $i$ be the involution acting on $\bar{V}$ that interchanges the sheets of the double cover $f$ and let $i_{\mathcal{E}}$ be the induced involution on $\mathcal{E} \times_{\bar{V}} i^{*} \mathcal{E}$. Assume the following genericity conditions are satisfied:

(1) Over the general point of $G$, the fiber of $\pi$ is irreducible and semistable.

(2) The image $f(G)$ contains no singular point of $D$, and $G \cap i(G)$ is finite.

Assume that $\left(\mathcal{E} \times_{\bar{V}} i^{*} \mathcal{E}\right) / i_{\mathcal{E}} \rightarrow \mathbb{P}^{2}$ admits a resolution of singularities $p: X \rightarrow \mathbb{P}^{2}$ which is an Abelian fibration on a projective hyper-Kähler manifold $X$ with a section $\tau$. Then $\bar{V} \cong \mathbb{P}^{1} \times \mathbb{P}^{1}$ and $X$ is birational to Hilb ${ }^{2}(S)$ for a projective elliptic K3 surface $S$ with a section.

Proof. Notice that the intersection $G \cap \tilde{D}$ consists of finitely many points because of the genericity conditions. Indeed, if $G \cap \tilde{D}$ wasn't finite, then $f(G)$ and $D$ would have a whole component in common and the fibers of $p$ above this component would be very degenerate and not semistable. More precisely, if $D$ is reducible, and $f(G)$ contains one of its irreducible components, then $f(G)$ would contain singular points of $D$, namely the points of intersection of the given irreducible component with other components, and this is a contradiction. On the other hand, if $D$ is irreducible, then $f(G)$ would contain $D$, i.e., $\tilde{D} \subset G$. Since $\tilde{D}$ is fixed by the involution $i$, we would get a contradiction with the condition that $G \cap i(G)$ is finite. This is the place where we use the "genericity conditions" (2).

Let $L \subset \mathbb{P}^{2}$ be a line intersecting $D$ transversally at general points of $D$ and avoiding the finite set $f(G \cap \tilde{D})=f(G) \cap D$. Denote the pre-image of $L$ in $\bar{V}$ by $L_{V}$. The restricted map $f_{V}: L_{V} \rightarrow L$ is 2:1. Since we have choosen $L$ to be general so that $L_{V}$ doesn't intersect $\tilde{D} \cap G$, then every fiber of $\mathcal{E}_{L_{V}} \times_{L_{V}} i^{*} \mathcal{E}_{L_{V}} \rightarrow L_{V}$ over a point of $L_{V} \cap \tilde{D}$ is smooth. After pulling back our construction to $L_{V}$, there is a rational map:

$$
L_{V} \times \mathbb{P}^{2} X \rightarrow \mathcal{E}_{L_{V}} \times_{L_{V}} i^{*} \mathcal{E}_{L_{V}}
$$

which by Weil's extension theorem [3, Theorem 4.4/1] is regular on the smooth locus of $L_{V} \times_{\mathbb{P}^{2}} X \rightarrow L_{V}$ since the fibers are abelian varieties.

Every section of $p: X \rightarrow \mathbb{P}^{2}$ is contained in the smooth locus of $X \rightarrow \mathbb{P}^{2}$, so it pulls back to a curve in the smooth locus of $L_{V} \times_{\mathbb{P}^{2}} X \rightarrow L_{V}$ via the section $i d_{L_{V}} \times\left._{L} \tau\right|_{L}$.

Since $\mathcal{K}_{X}=\mathcal{O}_{X}$, the relative dualizing sheaf of $p: X \rightarrow \mathbb{P}^{2}$ is $\omega_{X / \mathbb{P}^{2}}=p^{*} \mathcal{O}_{\mathbb{P}^{2}}(3)$, and therefore $\tau^{*} \omega_{X / \mathbb{P}^{2}}=\mathcal{O}_{\mathbb{P}^{2}}(3)$. The section $\sigma$ induces a section $(\sigma, \sigma \circ i)$ of the map:

$$
\operatorname{pr}_{\bar{V}}: \mathcal{E} \times_{\bar{V}} i^{*} \mathcal{E} \rightarrow \bar{V}
$$


Let $\tilde{\tau}: \bar{V} \rightarrow \bar{V} \times_{\mathbb{P}^{2}} X$ be the section of $\bar{V} \times_{\mathbb{P}^{2}} X \rightarrow \bar{V}$ obtained by base change from $\tau$. We consider $\tau f_{V}\left(L_{V}\right)$ as a section of the map $L_{V} \times_{\mathbb{P}^{2}} X \rightarrow L_{V}$ and in the notations above, it is $\tilde{\tau}\left(L_{V}\right)$. Consider the relative tangent bundle with the natural morphism:

$$
\left(\tau f_{V}\right)^{*} N_{\tilde{\tau}\left(L_{V}\right) / L \times_{\mathbb{P}^{2}} X}=\left.f_{V}^{*}\left(\tau^{*} T_{X / \mathbb{P}^{2}}\right) \rightarrow(\sigma, \sigma \circ i)^{*} T_{\mathcal{E} \times i^{*} \mathcal{E} / \bar{V}}\right|_{L_{V}}
$$

Since $L_{V} \times \mathbb{P}^{2} X$ is the blowup of $\mathcal{E}_{L_{V}} \times_{L_{V}} i^{*} \mathcal{E}_{L_{V}}$, by the behavior of the canonical class under blow-ups, we get the formula

$$
\left.\left.(\sigma, \sigma \circ i)^{*} \omega_{\mathcal{E} \times_{\bar{V}} i^{*} \mathcal{E} / \bar{V}}\right|_{L_{V}} \cong \tilde{\tau}^{*} \omega_{X / \mathbb{P}^{2}}\left(-L_{V} \cap \tilde{D}\right) \cong f^{*}\left[\mathcal{O}_{\mathbb{P}^{2}}(3)\left(-\frac{1}{2} D\right)\right]\right|_{L_{V}},
$$

and the last isomorphism holds because $f$ is $2: 1$.

However, $\omega_{\mathcal{E} \times \bar{V} i^{*} \mathcal{E} / \bar{V}}=\pi_{1}^{*} \omega_{\mathcal{E} / \bar{V}} \otimes \pi_{2}^{*} \omega_{i^{*} \mathcal{E} / \bar{V}}$, where $\pi_{1}$ and $\pi_{2}$ are the natural projections. Denote by $\overline{\mathcal{M}}_{1,1}$ the coarse moduli space of marked elliptic curves. The singular locus of a family of elliptic curves maps to the boundary of $\overline{\mathcal{M}}_{1,1}$. If we denote the pull-back of the boundary of $\overline{\mathcal{M}}_{1,1}$ by $\delta$, then it is well known that $\omega_{\mathcal{E} / \bar{V}}=\frac{\delta}{12}$ (see [6, Section 3.E]).

Therefore, using the classifying map from $L_{V}$ to the moduli space $\overline{\mathcal{M}}_{1,1}$, we obtain

$$
(\sigma, \sigma \circ i)^{*} \omega_{\mathcal{E} \times i^{*} \mathcal{E} / \bar{V}} \cong \mathcal{O}_{\bar{V}}\left(\frac{G+i^{-1} G}{12}\right)=\mathcal{O}_{\bar{V}}\left(\frac{f^{-1}(f(G))}{12}\right)=f^{*} \mathcal{O}_{\mathbb{P}^{2}}\left(\frac{f(G)}{12}\right)
$$

which is well-defined on $L_{V}$.

When we compare the isomorphisms (1) and (2), we get:

$$
\left.\left.f^{*} \mathcal{O}_{\mathbb{P}^{2}}\left(\frac{f(G)}{12}\right)\right|_{L_{V}} \cong f^{*}\left[\mathcal{O}_{\mathbb{P}^{2}}(3)\left(-\frac{1}{2} D\right)\right]\right|_{L_{V}}
$$

or equivalently,

$$
\left.\left.f^{*} \mathcal{O}_{\mathbb{P}^{2}}\left(\frac{D}{2}+\frac{f(G)}{12}\right)\right|_{L_{V}} \cong f^{*} \mathcal{O}_{\mathbb{P}^{2}}(3)\right|_{L_{V}}
$$

Comparing the degrees, we obtain the relation:

$$
\frac{1}{2} \operatorname{deg}(D)+\frac{1}{12} \operatorname{deg} f(G)=3
$$

The degrees of $D$ and $f(G)$ are positive even integers, hence there are two possibilities: $(\operatorname{deg}(D), \operatorname{deg}(G))=$ $(2,24)$ or $(4,12)$.

Case 1: $(\operatorname{deg}(D), \operatorname{deg}(f(G)))=(4,12)$

First we consider the case when $D$ is smooth. Since $\operatorname{deg}(D)=4, \bar{V}$ is a del Pezzo surface $\left(K_{\bar{V}}<0\right)$. We want to show that $\mathcal{E}$ is rationally connected. Take two general points $p, q \in \mathcal{E}$. Then $f(\pi(p)), f(\pi(q))$ are two general points in $\mathbb{P}^{2}$. Then $f(\pi(p)) \in L_{p}$, where $L_{p}$ is a tangent line to $D$ passing through $f(\pi(p))$, and $f(\pi(q)) \in L_{q}$, where $L_{q}$ is also a tangent line. Let $L_{p} \cap L_{q}=\{r\}$.

Take a tangent line $L$ to $D$ and pull it back to $\bar{V}: L_{\bar{V}} \doteq L \times_{\mathbb{P}^{2}} \bar{V}$. Its normalization is $\tilde{L}_{\bar{V}}$ and $\left.\mathcal{E}\right|_{\tilde{L}_{\bar{V}}}$ is an elliptic fibration over $\mathbb{P}^{1}$ with 12 nodal fibers. The surface is rational, because it is deformation equivalent to $\mathbb{P}^{2}$ blown-up at 9 points in the base locus of a pencil of plane cubics ([1, Section 5.12]).

We can lift the lines $L_{p}$ and $L_{q}$ to $\mathcal{E}$ and get $\left.\mathcal{E}\right|_{\tilde{L}_{p, \bar{V}}}$ and $\left.\mathcal{E}\right|_{\tilde{L}_{q, \bar{V}}}$ which are rational surfaces. We can connect any two points on a rational surface with a rational curve. Connect $p$ to $\tilde{r}$ and $q$ to $\tilde{r}$, where $\tilde{r} \in(f \pi)^{-1}(r)$, so $p$ is rationally chain connected to $q$.

The point $\tilde{r}$ is a smooth point of $\mathcal{E}$. In characteristic 0 , we can smooth the nodal rational curve if we have a general pair $p, q$ and if $\tilde{r}$ is smooth ([4, Section 4.6]). Therefore, $\mathcal{E}$ is rationally connected. 
Fix a section $s$ of $i^{*} \mathcal{E} \rightarrow \bar{V}$. Then we get a section $\tilde{s}$ of $\mathcal{E} \times_{\bar{V}} i^{*} \mathcal{E} \rightarrow \mathcal{E}$. Since we have a finite morphism $\mathcal{E} \times_{\bar{V}} i^{*} \mathcal{E} \rightarrow X$, we get a finite morphism $\mathcal{E} \rightarrow X$.

The image of a finite morphism from a rationally connected variety to a hyper-Kähler manifold is of dimension at most $\frac{1}{2} \operatorname{dim}(X)$, because we have a $(2,0)$ form on $X$ and there are no holomorphic $(2,0)$ or $(1,0)$ forms on a rational variety. However, $\operatorname{dim}(\mathcal{E})=3$ and it is bigger than $\frac{1}{2} \operatorname{dim}(X)=2$ - a contradiction.

Now assume $D$ is singular. Consider the linear system of lines $l$ containing a fixed singular point $r$ of $D$. Consider the associated linear system $f^{-1}(l)$. These divisors will typically be all singular because $\bar{V}$ is singular at $f^{-1}(r)$. However, the linear system of strict transforms $\widetilde{f^{-1}(l)}$ on $B l_{f^{-1}(r)} \bar{V}$ is a basepoint free pencil of divisors on the normal surface $B l_{f^{-1}(r)} \bar{V}$. Thus, by Bertini's theorem, a general member of this pencil is smooth and intersects $G$ transversally. In particular, the surface $\mathcal{E}_{f_{f^{-1}(l)}}$ is smooth for such a member.

Let $L$ be any line passing through a singular point $r \in D$. Every component of $f^{-1}(L)$ is a rational curve. Without loss of generality we shall consider the irreducible case. The normalization $\widetilde{f^{-1}(L)}$ is isomorphic to $\mathbb{P}^{1}$. Therefore, $\left.\mathcal{E}\right|_{\widetilde{f^{-1}(L)}}$ is an elliptic surface over $\mathbb{P}^{1}$. Since there is the following relation of intersection numbers: $f^{-1}(L) \cdot G=L \cdot f(G)=12$, it follows that $\left.\mathcal{E}\right|_{f^{-1}(L)}$ is a rational surface.

Consider the 1-parameter family $\mathcal{F}$ of such rational surfaces parametrized by $\mathbb{P}^{1}$ (since the line $L$ varies in $\left.\mathbb{P}^{1}\right)$. The 3 -fold $\mathcal{F}$ is rationally connected inside a 4 -dimensional hyper-Kähler manifold which is impossible, because $\operatorname{dim}(\mathcal{F})>\frac{1}{2} \operatorname{dim}(X)$ and there are no holomorphic $(2,0)$ forms on $\mathcal{F}$.

We ruled out the first case completely and the only remaining case is:

Case 2: $(\operatorname{deg}(D), \operatorname{deg}(f(G)))=(2,24)$

First we consider the case when $D$ is smooth. Then $\bar{V} \cong \mathbb{P}^{1} \times \mathbb{P}^{1}$.

Take a tangent line $L$ to the conic and pull it back to $\bar{V}: L_{\bar{V}} \doteq L \times_{\mathbb{P}^{2}} \bar{V}$. Its normalization $\tilde{L}_{\bar{V}}$ is reducible and consists of two copies of $\mathbb{P}^{1}$, say $\tilde{L}_{\bar{V}, 1}$ and $\tilde{L}_{\bar{V}, 2}$.

\section{Case 2.1:}

$\left.\mathcal{E}\right|_{\tilde{L}_{V, k}}$ is an elliptic fibration over $\mathbb{P}^{1}$ with 12 nodal fibers, $k=1$, 2. Then we repeat the same argument as in Case 1 in order to exclude this case.

\section{Case 2.2:}

$\left.\mathcal{E}\right|_{\tilde{L}_{\bar{V}, 1}}$ is an elliptic fibration over $\mathbb{P}^{1}$ with 24 nodal fibers and $\left.\mathcal{E}\right|_{\tilde{L}_{\bar{V}, 2}}$ is an elliptic fibration over $\mathbb{P}^{1}$ with no singular fibers. Then $\left.\mathcal{E}\right|_{\tilde{L}_{\bar{V}, 2}}$ is the trivial fibration and therefore, $\mathcal{E}$ is the pull back of an elliptic fibration on $\mathbb{P}^{1}$ through the projection on this factor. And, since the elliptic fibration on $\mathbb{P}^{1}$ has 24 nodal fibers, it is an elliptic K3 surface $S \rightarrow \mathbb{P}^{1}$.

After considering all the cases, we see that:

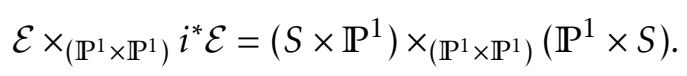

We want to prove that $\left(S \times \mathbb{P}^{1}\right) \times\left(\mathbb{P}^{1} \times \mathbb{P}^{1}\right)\left(\mathbb{P}^{1} \times S\right) \cong S \times S$. Indeed, we have the following commutative fiber diagram:

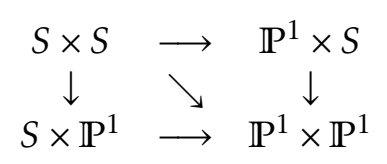

Therefore,

$$
\mathcal{E} \times\left(\mathbb{P}^{1} \times \mathbb{P}^{1}\right) i^{*} \mathcal{E}=\left(S \times \mathbb{P}^{1}\right) \times_{\left(\mathbb{P}^{1} \times \mathbb{P}^{1}\right)}\left(\mathbb{P}^{1} \times S\right) \cong S \times S .
$$




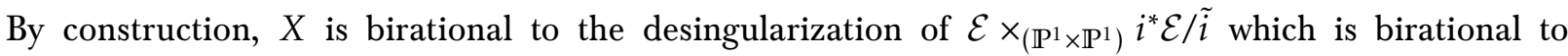
$S \times S / \mathbb{Z}_{2}$. Therefore, $X$ is birational to the desingularization of $S \times S / \mathbb{Z}_{2}$ which is $\operatorname{Hilb}^{2}(S)$ by Fogarty's theorem (Theorem 2.6).

Now consider the case when $D$ is singular. Since $\bar{V}$ is normal, $D$ cannot be a double line. Therefore, $D$ is the union of two lines and $\bar{V}$ is a singular quadric cone.

Let $L$ be a line in $\mathbb{P}^{2}$ passing through the node $r \in D$. Then $f^{-1}(L)$ is the union of two lines $L_{1}$ and $L_{2}$ each one of which is a line in the cone $\bar{V}$ passing through its vertex. On a quadric cone all lines are algebracially equivalent, and in particular $L_{1}$ and $L_{2}$ are algebraically equivalent. Since $\operatorname{deg}(G)=24$, $L_{1} \cdot G=L_{2} \cdot G=12$. Therefore, the surface $\left.\mathcal{E}\right|_{L_{i}}$ is rational (for $i=1,2$ ). We constructed a rationally parametrized 1-parameter family of rational surfaces which cannot exist in a 4-dimensional hyper-Kähler manifold. With this we finish the proof of our main theorem.

Acknowledgements. The author is thankful to Prof. G. Tian for asking questions leading to this problem. She thanks the referees for their helpful suggestions and for improving the exposition of the paper. The author is grateful to the Institute for Advanced Study for their hospitality.

\section{References}

[1] W. Barth, K. Hulek, C. Peters, and A. Van de Ven, Compact complex surfaces. Second edition. Ergebnisse der Mathematik und ihrer Grenzgebiete. 3. Folge. A Series of Modern Surveys in Mathematics, vol. 4, Springer-Verlag, Berlin, 2004. MR-2030225

[2] A. Beauville, Variétés Kähleriennes dont la première classe de Chern est nulle, J. Differential Geom. 18 (1983), no. 4, 755-782. MR-0730926

[3] S. Bosch, W. Lütkebohmert, and M. Raynaud, Néron models, Ergebnisse der Mathematik und ihrer Grenzgebiete (3), vol. 21, Springer-Verlag, Berlin, 1990. MR-1045822

[4] O. Debarre, Higher-dimensional algebraic geometry, Universitext, Springer-Verlag, New York, 2001. MR1841091

[5] J. Fogarty, Algebraic families on an algebraic surface, Amer. J. Math. 90 (1968), 511-521. MR-0237496

[6] J. Harris and I. Morrison, Moduli of curves, Graduate Texts in Mathematics, vol. 187, Springer-Verlag, New York, 1998. MR-1631825

[7] D. Huybrechts, Compact hyper-Kähler manifolds: basic results, Invent. Math. 135 (1999), no. 1, 63-113. MR-1664696

[8] J.-M. Hwang, Base manifolds for fibrations of projective irreducible symplectic manifolds, Invent. Math. 174 (2008), no. 3, 625-644. MR-2453602

[9] D. Markushevich, Lagrangian families of Jacobians of genus 2 curves, J. Math. Sci. 82 (1996), no. 1, 3268-3284. MR-1423641

[10] D. Matsushita, On fibre space structures of a projective irreducible symplectic manifold, Topology 38 (1999), no. 1, 79-83. MR-1644091

[11] D. Matsushita, Higher direct images of dualizing sheaves of Lagrangian fibrations, Amer. J. Math. 127 (2005), no. 2, 243-259. MR-2130616

[12] K. O'Grady, Desingularized moduli spaces of sheaves on a K3, J. Reine Angew. Math. 512 (1999), 49-117. MR-1703077 
[13] K. O’Grady, A new six-dimensional irreducible symplectic variety, J. Algebr. Geom. 12 (2003), no. 3, 435-505. MR-1966024

[14] B. Wieneck, On polarization types of Lagrangian fibrations, Manuscripta Math. 151 (2016), no. 3-4, 305327. MR-3556822

[15] B. Wieneck, Monodromy invariants and polarization types of generalized Kummer fibrations, Math. Z. 290 (2018), no. 1-2, 347-378. MR-3848435 